\title{
Pathophysiological implications of actin-free Gc-globulin concentration changes in blood plasma and cerebrospinal fluid collected from patients with Alzheimer's disease and other neurological disorders
}

\author{
Alina Kułakowska,D, Joanna Tarasiuk 1,D, Katarzyna Kapica-Topczewska ${ }^{1, D}$, Monika Chorąży 1,D, \\ Robert Pogorzelski ${ }^{1, D}$, Agnieszka Kulczyńska-Przybik', ${ }^{2, A}$, Barbara Mroczko ${ }^{2, A}$, Robert Bucki ${ }^{3, D}$ \\ ${ }^{1}$ Department of Neurology, Medical University of Bialystok, Poland \\ ${ }^{2}$ Department of Neurodegeneration Diagnostics, Medical University of Bialystok, Poland \\ ${ }^{3}$ Department of Microbiological and Nanobiomedical Engineering, Medical University of Bialystok, Poland \\ A - research concept and design; B - collection and/or assembly of data; $\mathrm{C}$ - data analysis and interpretation; \\ $D$ - writing the article; $E$ - critical revision of the article; $F$ - final approval of the article
}

Address for correspondence

Joanna Tarasiuk

E-mail:amirtarasiuk@wp.pl

\section{Funding sources}

This work was supported by the National Science Center (Poland) with grant No. UMO-2015/17/B/ NZ6/03473 (to RB) and Medical University of Bialystok grant No. N/ST/2B/16/002/1144 (to AK).

Conflict of interest

None declared

Received on December 10, 2016

Reviewed on April 7, 2017

Accepted on April 12, 2017

DOI

10.17219/acem/70441

Copyright

Copyright by Author(s)

This is an article distributed under the terms of the

Creative Commons Attribution Non-Commercial License

(http://creativecommons.org/licenses/by-nc-nd/4.0/)

\begin{abstract}
Background. The extracellular actin scavenging system (EASS) is composed of plasma Gc-globulin and gelsolin, and is responsible for the elimination of toxic actin from the bloodstream.

Objectives. In this study, we assessed the actin-free Gc-globulin concentrations in blood plasma and cerebrospinal fluid (CSF) obtained from subjects with neurodegenerative and inflammatory diseases of the central nervous system (CNS) as well as in a control group.
\end{abstract}

Material and methods. Using an enzyme-linked immunosorbent assay (ELISA), we measured the actinfree Gc-globulin concentrations in blood plasma and CSF obtained from subjects diagnosed with Alzheimer's disease (AD) ( $n=20)$, amyotrophic lateral sclerosis (ALS) $(n=12)$, multiple sclerosis (MS) $(n=42)$, tick-borne encephalitis (TBE) $(n=12)$, and from a control group $(n=20)$.

Results. The concentrations of free Gc-globulin in plasma collected from patients diagnosed with AD $(509.6 \pm 87.6 \mathrm{mg} / \mathrm{L})$ and ALS $(455.5 \pm 99.8 \mathrm{mg} / \mathrm{L})$ did not differ significantly between each other, but were significantly higher compared to the reference group $(311.7 \pm 87.5 \mathrm{mg} / \mathrm{L})(\mathrm{p}<0.001$ and $p<0.006$, respectively) as well as to MS $(310.8 \pm 66.6 \mathrm{mg} / \mathrm{L})(p<0.001$ and $p<0.001$, respectively) and TBE $(256.7 \pm 76 \mathrm{mg} / \mathrm{L})(p<0.001$ and $p<0.003$, respectively). In CSF collected from patients diagnosed with $A D$ and $A L S$, the concentrations of free $G c-g l o b u l i n$ were $2.6 \pm 1.1 \mathrm{mg} / \mathrm{L}$ and $2.7 \pm 1.9 \mathrm{mg} / \mathrm{L}$, respectively. They did not differ significantly between each other and were significantly higher compared to the reference group $(1.5 \pm 0.9 \mathrm{mg} / \mathrm{L})(\mathrm{p}<0.005$ and $p<0.041$, respectively). Interestingly, in patients with AD, significantly higher values of $\mathrm{Gc}$-globulin were detected compared to MS patients $(1.7 \pm 0.9 \mathrm{mg} / \mathrm{L})(\mathrm{p}<0.013)$.

Conclusions. Higher concentrations of free Gc-globulin in blood plasma and CSF collected from patients suffering from neurodegenerative diseases may indicate a potential role of this protein in their pathogenesis, and represent a potential tool for the diagnosis of CNS diseases.

Key words: Alzheimer's disease, amyotrophic lateral sclerosis, multiple sclerosis, tick-borne encephalitis, Gc-globulin 


\section{Introduction}

Gc-globulin (vitamin D binding protein) is a multifunctional, monomeric glycoprotein belonging to the $\alpha 2$ globulin fraction. This peptide is composed of 458 amino acids with 3 domains and has a molecular weight of 51-58 kDa. Gc-globulin is mainly produced by the liver, but it is also found in the kidneys, lungs, heart, spleen, and brain, as well as in several body fluids, including blood plasma, saliva, semen, breast milk, and cerebrospinal fluid (CSF). ${ }^{1}$ The serum concentration of free Gc-globulin (actin-free Gc-globulin) ranges between 92 and $332 \mathrm{mg} / \mathrm{L} .^{2}$ The laboratory standard for the Gc-globulin concentration in CSF has not been established.

The primary function of Gc-globulin is the removal of actin. Circulating G-actin, released from injured tissues, polymerizes and forms F-actin filaments promoting certain disorders, including disseminated intravascular coagulation (DIC). ${ }^{1,2} \mathrm{~F}$-actin filaments are depolymerized by gelsolin releasing $\mathrm{G}$-actin monomers, which are subsequently bound to Gc-globulin. The G-actin-gelsolin and G-actin-Gc-globulin complexes are primarily removed from the circulation by mononuclear phagocytes. ${ }^{3,4}$ Low concentrations of Gc-globulin correspond to a poor prognostic outlook in acute liver failure, multiple organ dysfunction syndrome and sepsis. ${ }^{5,6}$ Gc-globulin is also involved in the transport of vitamin $\mathrm{D}$ with its metabolites, fatty acids and endotoxins, functions in the activation of osteoclasts and macrophages, and serves as a chemotactic cue for leukocytes. ${ }^{1,2,7}$

Cerebrospinal fluid provides valuable information about biochemical changes in the central nervous system (CNS); the examination of CSF is an important tool in the diagnosis of CNS diseases. ${ }^{8}$ Pathological CNS processes are reflected in the protein composition of CSF. Information on the concentration of free Gc-globulin in the blood and CSF of patients with neurodegenerative diseases is limited.

The aim of the current study was to measure and compare the concentration of free Gc-globulin in the serum and CSF of patients with: 1. neurodegenerative diseases, including Alzheimer's disease (AD) and amyotrophic lateral sclerosis (ALS); 2. diseases associated with inflammatory and neurodegenerative etiopathogenesis, such as multiple sclerosis (MS); 3. infectious inflammatory disease, e.g., tick born encephalitis (TBE); 4. and in a control group consisting of patients suffering from conditions which do not alter the standard parameters of CSF, such as idiopathic headache and idiopathic facial nerve palsy.

\section{Material and methods}

\section{Patients and the preparation of samples}

Blood and CSF samples were obtained from patients admitted to the Department of Neurology and the Department of Infectious Diseases and Neuroinfections of The Medical University of Białystok Clinical Hospital, Poland. The study was approved by the Medical University of Bialystok Ethics Committee for Research on Humans and Animals (R-I-002/382/2012) and written consent was obtained from all subjects. All individuals underwent lumbar puncture for diagnostic purposes. The clinical characteristics of patients are shown in Table 1.

The diagnosis of AD was based on the criteria of the National Institute of Neurologic, Communicative Disorders and Stroke/Alzheimer's Disease and Related Disorders Association (NINCDS-ADRDA). ${ }^{9,10}$ All patients had memory loss and impaired cognitive function lasting more than 6 months validated using psychological tests and biochemical assessment (Table 2).

The relapsing-remitting MS diagnosis was based on the McDonald criteria. ${ }^{11}$ The degree of disability of MS patients was assessed using the Expanded Disability Status Scale (EDSS), with a mean score of $1.5 \pm 0.6$ indicating the early stage of disease. ${ }^{12}$ All patients underwent head magnetic resonance imaging (MRI) examination, which demonstrated multiple disseminated demyelinating

Table 1. Patient clinical characteristics

\begin{tabular}{|c|c|c|c|c|c|c|}
\hline \multicolumn{5}{|c|}{ Clinical characteristics of patients } & \multicolumn{2}{|c|}{ CSF } \\
\hline diagnosis & $\begin{array}{c}\text { number of patients } \\
\text { (women) }\end{array}$ & age [years] & EDSS & Q Alb & $\begin{array}{l}\text { total protein } \\
{[\mu \mathrm{g} / \mathrm{mL}]}\end{array}$ & $\begin{array}{l}\text { lymphocytes } \\
(0-5 \text { cells } / \mu \mathrm{L})\end{array}$ \\
\hline$A D$ & $20(13)$ & $69.9 \pm 10.4$ & - & $5.7 \pm 1.3$ & $38.3 \pm 17.2$ & $1.25 \pm 1.0$ \\
\hline ALS & $12(9)$ & $57.5 \pm 10.3$ & - & $6.1 \pm 2.3$ & $39.7 \pm 15.3$ & $2.2 \pm 2.3$ \\
\hline$M S^{*}$ & $42(25)$ & $35.5 \pm 10.5$ & $1.5 \pm 0.5$ & $6.3 \pm 1.5$ & $375 \pm 125$ & $3.8 \pm 2.1$ \\
\hline TBE & $12(3)$ & $53.1 \pm 24.3$ & - & $12.4 \pm 1.2^{* *}$ & $882 \pm 157^{* *}$ & $110 \pm 15.6^{* *}$ \\
\hline \multicolumn{7}{|c|}{ Reference group $(n=20)$} \\
\hline Idiopathic headache & $13(11)$ & $40.2 \pm 20.4$ & - & $6.5 \pm 1.3$ & $409 \pm 155$ & $3.0 \pm 2.1$ \\
\hline $\begin{array}{l}\text { Idiopathic facial } \\
\text { nerve palsy }\end{array}$ & $7(5)$ & $48.4 \pm 15.3$ & - & $7.5 \pm 0.6$ & $357 \pm 179$ & $5.1 \pm 2.1$ \\
\hline
\end{tabular}

CFS - cerebrospinal fluid; AD - Alzheimer's disease; ALS - amyotrophic lateral sclerosis; MS - multiple sclerosis; TBE - tick-borne encephalitis; Q Alb - coefficient of albumin; EDSS - Expanded Disability Status Scale; * all patients with MS in CSF presented oligoclonal bands of IgG (type 2 or 3); ** incorrect values. 
plaques. None of the patients were treated with corticosteroids or immunomodulating drugs (beta-interferon, glatiramer acetate, natalizumab).

The clinically definite ALS was diagnosed using the Airlie House/El Escorial Revisited World Federation of Neurology criteria. ${ }^{13,14}$ All patients with ALS showed features of damage to upper and lower motor neurons as confirmed by electromyography (EMG) examination. None of the patients were treated with riluzol.

The diagnosis of TBE was confirmed by the detection of antibodies against the TBE virus in serum and CSF by enzyme-linked immunosorbent assay (ELISA) tests (Virion-Serion Kit; SERION ${ }^{\circledR}$ Immunologics, Würzburg, Germany). All patients with TBE received symptomatic treatment with no corticosteroids.

The reference group consisted of patients with idiopathic headache and idiopathic facial nerve palsy (Bell's palsy), with no active inflammatory process. None of the patients with idiopathic facial nerve palsy were immunocompromised or had herpes simplex virus 1 antibodies in blood as measured by ELISA (ELISA kit; Genzyme Virotech $\mathrm{GmbH}$, Rüsselsheim, Germany).

The samples of anticoagulated blood were centrifuged and the collected plasma was frozen at $-80^{\circ} \mathrm{C}$. After collection, CSF underwent a standard examination. The CSF samples were then centrifuged ( $2000 \times \mathrm{g}, 20 \mathrm{~min})$ and the supernatants were subjected to total protein analysis and frozen at $-80^{\circ} \mathrm{C}$. CSF analysis included physical properties, cytosis, the total protein concentration and Q Alb ratio $(\mathrm{Q} A l b=\mathrm{al}-$ bumin in CSF $[\mathrm{mg}] /$ serum albumin $[\mathrm{g}] \times 1000$ ) indicating the efficiency of the blood-CSF barrier. In patients with clinically probable AD, we assessed the average concentration of $A \beta 1-42, A \beta 1-40$, the $A \beta 1-42 / A \beta 1-40$ ratio and the average concentration of Tau and phosphorylated Tau (pTau) protein using the ELISA INNOTEST ${ }^{\circledR}$ kits (Innogenetics GmbH, Hannover, Germany) and ELISA Kits (IBL International $\mathrm{GmbH}$, Hamburg, Germany). The average values of $A \beta 1-42, A \beta 1-40$, the $A \beta 1-42 / A \beta 1-40$ ratio, Tau, and $p$ Tau were typical for patients with AD (Table 2).

The Gc-globulin actin-free ELISA Kit from BioPorto Diagnostics (Hellerup, Denmark) was used to assess free Gc-globulin.

\section{Statistical analysis}

The results were analyzed statistically using the KruskalWallis test followed by post hoc analysis with the DwassSteel-Critchlow-Fligner test. A p-value $<0.05$ was considered statistically significant.

\section{Results}

Our results show significantly higher concentrations of free Gc-globulin in the serum and CSF of patients with neurodegenerative diseases compared to either the reference group or patients with inflammatory disease. The concentration of free Gc-globulin in the serum of patients with $\mathrm{AD}(509.6 \pm 87.6 \mathrm{mg} / \mathrm{L})$ or ALS $(455.5 \pm 99.8 \mathrm{mg} / \mathrm{L}) \mathrm{did}$ not differ significantly; however, in both groups, these concentrations were significantly higher than in the reference group $(311.7 \pm 87.5 \mathrm{mg} / \mathrm{L})(\mathrm{p}<0.001$ and $\mathrm{p}<0.006$, respectively). This is in agreement with previous studies demonstrating that the concentrations of free Gc-globulin in the serum of patients suffering from neurodegenerative diseases were greater than the values reported for healthy subjects. ${ }^{2}$ Furthermore, the concentration of free Gc-globulin in the serum of patients with AD or ALS was also higher than in subjects with inflammatory diseases, such as TBE $(256.7 \pm 76 \mathrm{mg} / \mathrm{L})(\mathrm{p}<0.001$ and $\mathrm{p}<0.003$, respectively) and MS $(310.8 \pm 66.6 \mathrm{mg} / \mathrm{L})(\mathrm{p}<0.001$ and $\mathrm{p}<0.001$, respectively) (Fig. 1).

The standard parameters for CSF evaluation were representative of the studied diseases (AD, ALS) and are presented in Table 1. The concentration of free Gc-globulin in CSF displayed trends similar to those observed

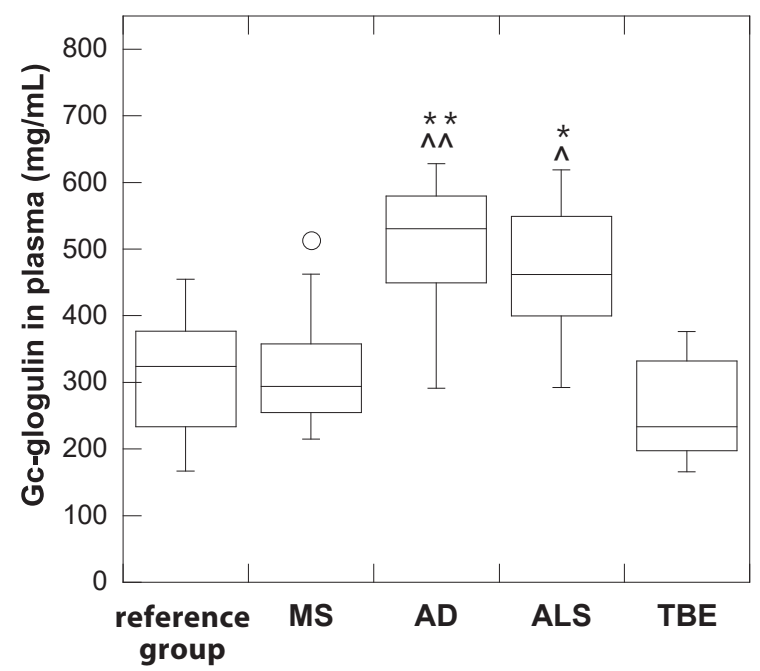

Fig. 1. Comparison of the free Gc-globulin concentration in blood plasma

${ }^{*} p<0.006 ;{ }^{* *} p<0.001$ compared to the reference group; $\wedge p<0.003$

$\wedge \wedge p<0.001$ compared to patients with tick-borne encephalitis;

${ }^{\circ} \mathrm{p}<0.001$ compared to patients with multiple sclerosis; AD - Alzheimer's

disease; ALS - amyotrophic lateral sclerosis; MS - multiple sclerosis; TBE - tick-borne encephalitis; the Kruskal-Wallis test with post hoc Dwass-Steele-Critchlow-Fligner test.

Table 2. Biomarkers of neurodegeneration in patients with Alzheimer's disease

\begin{tabular}{|c|c|c|c|}
\hline $\begin{array}{c}A \beta 1-42 \\
\text { (cut-off }=590 \mathrm{pg} / \mathrm{mL} \text { ) }\end{array}$ & $\begin{array}{c}\text { Tau } \\
\text { (cut-off }=300 \mathrm{pg} / \mathrm{mL} \text { ) }\end{array}$ & $\begin{array}{c}\text { pTau } \\
\text { (cut-off }=50 \text { pg/mL) }\end{array}$ & $\begin{array}{l}\text { Ratio } A \beta 1-42 / A \beta 1-40 \\
\quad(\text { cut-off }=0.030)\end{array}$ \\
\hline $466.45 \pm 296.65$ & $353.35 \pm 176.13$ & $55.2 \pm 21.67$ & $0.0251 \pm 0.014$ \\
\hline
\end{tabular}


for serum. The concentration of free Gc-globulin in patients with $\operatorname{AD}(2.6 \pm 1.1 \mathrm{mg} / \mathrm{L})$ and ALS $(2.7 \pm 1.9 \mathrm{mg} / \mathrm{L})$ did not differ significantly between each other, but were significantly higher compared to the reference group $(1.5 \pm 0.9 \mathrm{mg} / \mathrm{L})(\mathrm{p}<0.005$ and $\mathrm{p}<0.041$, respectively). The average concentrations of free Gc-globulin in the CSF of MS $(1.7 \pm 0.9 \mathrm{mg} / \mathrm{L})$ and TBE $(1.8 \pm 1.2 \mathrm{mg} / \mathrm{L})$ patients were not significantly different than in the case of the reference group. However, there was observed a significant difference between the concentrations of free Gc-globulin in the CSF of patients with AD and MS ( $<$ < 0.013) (Fig. 2).

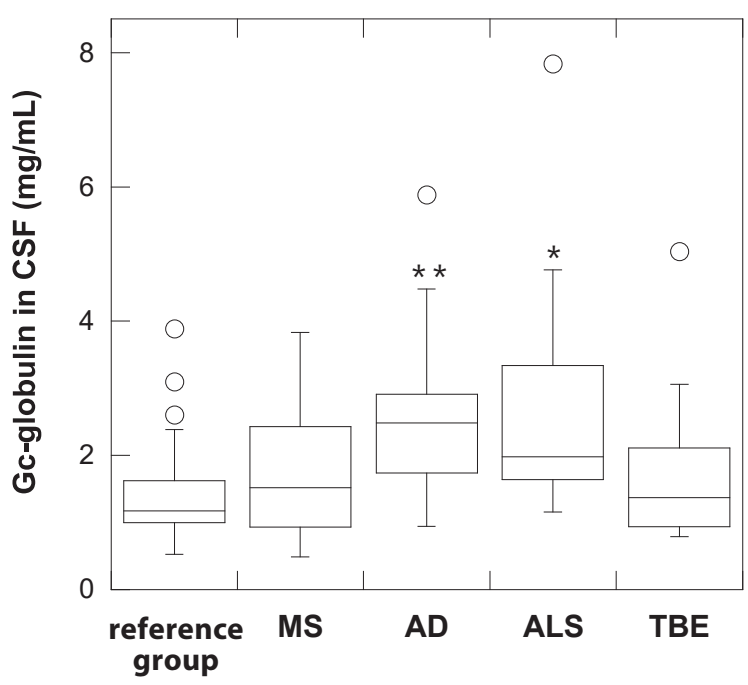

Fig. 2. Comparison of the free Gc-globulin in cerebrospinal fluid (CSF)

${ }^{*} \mathrm{p}<0.041$; $^{* *} \mathrm{p}<0.005$ compared to the reference group; ${ }^{\circ} \mathrm{p}<0.013$ compared to patients with multiple sclerosis; AD - Alzheimer's disease; ALS - amyotrophic lateral sclerosis; MS - multiple sclerosis; TBE - tickborne encephalitis; the Kruskal-Wallis test with post hoc Dwass-SteeleCritchlow-Fligner test.

\section{Discussion}

There are few reports concerning the concentration and function of free Gc-globulin in the pathogenesis of neurodegenerative diseases. Our earlier studies indicate that there are no significant differences between the Gc-globulin concentrations in the plasma and CSF of MS patients compared to control subjects. ${ }^{4}$ In a previous study on patients with dementive diseases, including AD, Parkinson's disease and Pick's disease, the Gc-globulin concentration was higher compared to patients with head trauma, autoimmune or cerebrovascular disorders. ${ }^{15}$ Furthermore, Zhang et al., using a multiplex analysis of CSF proteins, demonstrated a statistically significant increase in the concentration of free Gc-globulin in the CSF of patients with AD and Parkinson's disease compared to a group of healthy controls. ${ }^{16}$

Increased levels of Gc-globulin in the serum and CSF of patients with AD and ALS may be associated with neurodegeneration in the CNS. The breakdown of neurons has been described for both AD and ALS. In AD, this process primarily occurs in the cerebral cortex/hippocampus and for ALS - in the upper and lower motor neurons. Actin released from damaged tissue is removed from blood and purportedly from CSF through the extracellular actin scavenging system (EASS). ${ }^{17-20}$ It is likely that the chronic nature of neurodegenerative disease initiates a compensatory increase in the hepatic and intrathecal synthesis of Gcglobulin as a response to elevated levels of actin. In our patients with AD and ALS, the high concentration of free Gc-globulin in CSF may be due to the increased neuronal synthesis, because the patients were at an early stage of the disease and there was no disruption of the bloodCSF barrier, as indicated by Q Alb (Table 1).

Alternatively, the increased concentration of free Gcglobulin observed in both the serum and CSF of patients suffering from neurodegenerative diseases may indicate impaired G-actin binding. G-actin is released from F-actin filaments through the severing activity of gelsolin. Previous studies report reduced gelsolin expression in both the choroid plexus and CSF from AD patients. ${ }^{21}$ Effectively, the dysfunction of the EASS may switch on compensatory mechanisms, including increased Gc-globulin expression. Moreover, gelsolin is a $\mathrm{Ca}^{2+} /$ phosphatidylinositol 4,5-bisphosphate $\left(\mathrm{PIP}_{2}\right)$-regulated protein. Its $\mathrm{PIP}_{2}$ binding domain interacts with several acidic lipid signaling molecules, including $\mathrm{PIP}_{2}$, lysophosphatidic acid (LPA), lipoteichoic acid (LTA), lipopolysaccharide (LPS), sphingosine 1-phosphate (S1P), and its synthetic structural analogue fingolimod (FTY720P). ${ }^{22-25}$ When complexed with bioactive lipids, gelsolin loses the ability to bind and sever actin filaments as well as alters the ability of the lipids to function as cell agonists. In AD, inflammatory processes such as activation of microglia and astrocytes as well as the production of inflammatory cytokines occur within and around amyloid plaques. ${ }^{26}$ Alterations to the sphingolipids profile are linked to the pathogenesis of AD, and fingolimod seems to be a promising therapeutic agent reducing the accumulation of amyloid $\beta .{ }^{27-30}$ Taken together, this data suggests that, during the course of the neurodegenenerative process, the association of gelsolin with bioactive sphingolipids leads to a reduction in its ability to depolymerize F-actin and to prevent the release of G-actin monomers. As a consequence, elevated levels of unbound, free Gc-globulin may be observed.

Neurodegeneration also plays an important role in the pathogenesis of MS, but inflammatory processes dominate the early stages of disease. ${ }^{31-35}$ All the patients included in this study were in the early stage of the disease (EDSS of $1.5 \pm 0.5$ ); diagnostic lumbar puncture confirmed this diagnosis. Therefore, the results obtained from these patients and from patients with TBE, a representative disease of infectious inflammatory etiology, are very similar. In inflammatory processes, lower concentrations of free Gc-globulin in serum and CSF may result from the fact that inflammation proceeds more rapidly compared to neurodegeneration. ${ }^{36}$ It is possible that the rapid 
inflammatory process causes excessive cell destruction, causing high Gc-globulin consumption and insufficient activation of compensatory pathways.

In addition to actin scavenging, Gc-globulin plays a crucial role in vitamin D metabolism. ${ }^{1,2}$ In recent years, vitamin $\mathrm{D}$ has attracted much attention, and there is growing evidence that beyond its physiologic effects on calcium/ phosphorus homeostasis, low vitamin D status is associated with several diseases, including cancer, diabetes mellitus, rheumatoid arthritis, and other autoimmune conditions. ${ }^{37,38}$ Recently, vitamin D deficiency has been shown to be a risk factor for dementia and AD. ${ }^{39}$ However, it seems rather unlikely that altered levels of vitamin D affect the concentrations of Gc-globulin. In physiological conditions, only $5 \%$ of total plasma Gc-globulin is occupied by vitamin D and the plasma clearance of Gc-globulin does not appear to be altered by vitamin D binding.,

In conclusion, the results of our preliminary studies suggest that significantly higher concentrations of free Gcglobulin in serum and CSF in neurodegenerative diseases may result from their pathogenesis. Since neurodegeneration begins much earlier than the appearance of clinical signs, determining the concentration of certain proteins involved in its pathogenesis, such as Gc-globulin, may be important in the early diagnosis of CNS disease.

\section{References}

1. White $\mathrm{P}$, Cooke $\mathrm{N}$. The multifunctional properties and characteristics of vitamin D-binding protein. Trends Endocrinol Metab. 2000;11:320-327.

2. Meier U, Gressner O, Lammert F, Gressner AM. Gc-globulin: Roles in response to injury. Clin Chem. 2006;52:1247-1253.

3. Kułakowska A, Zajkowska JM, Ciccarelli NJ, Mroczko B, Drozdowski W, Bucki R. Depletion of plasma gelsolin in patients with tickborne encephalitis and Lyme neuroborreliosis. Neurodegener Dis. 2011;8:375-380.

4. Kułakowska A, Ciccarelli NJ, Wen Q, et al. Hypogelsolinemia, a disorder of the extracellular actin scavenger system, in patients with multiple sclerosis. BMC Neurol. 2010;10:107.

5. Schiødt FV, Bangert K, Shakil AO, et al. Predictive value of actin-free Gc-globulin in acute liver failure. Liver Transpl. 2007;13:1324-1329.

6. Bagchi A, Kumar S, Ray PC, Das BC, Gumma PK, Kar P. Predictive value of serum actin-free Gc-globulin for complications and outcome in acute liver failure. J Viral Hepat. 2015;22:192-200.

7. Łukaszewicz-Zajac M, Mroczko B, Kułakowska A, Szmitkowski M. The significance of Gc-globulin in clinical practice [in Polish]. Postepy Hig Med Dosw. 2008;62:625-631.

8. Bredesen DE, Rao RV, Mehlen P. Cell death in the nervous system. Nature. 2006;443:796-802.

9. Dubois B, Feldman HH, Jacova C, et al. Research criteria for the diagnosis of Alzheimer's disease: Revising the NINCDS-ADRDA criteria. Lancet Neurol. 2007;6:734-746.

10. Croisile B, Auriacombe S, Etcharry-Bouyx F, Vercelletto M; NloA \& Alzheimer's Association. The new 2011 recommendations of the National Institute on Aging and the Alzheimer's Association on diagnostic guidelines for Alzheimer's disease: Preclinical stages, mild cognitive impairment, and dementia [in French]. Rev Neurol (Paris). 2012;168:471-482.

11. Polman CH, Reingold SC, Banwell B, et al. Diagnostic criteria for multiple sclerosis: 2010 revisions to the McDonald criteria. Ann Neurol. 2011;69:292-302.

12. Kurtzke JF. Rating neurologic impairment in multiple sclerosis: An expanded disability status scale (EDSS). Neurology. 1983;3:1444-1452.
13. Brooks BR, Miller RG, Swash M, Munsat TL; World Federation of Neurology Research Group on Motor Neuron Disease. El Escorial revisited: Revised criteria for the diagnosis of amyotrophic lateral sclerosis. Amyotroph Lateral Scler Other Motor Neuron Disord. 2000;1:293-299.

14. Chieia MA, Oliveira AS, Silva HC, Gabbai AA. Amyotrophic lateral sclerosis: Considerations on diagnostic criteria. Arq Neuropsiquiatr. 2010;68:837-842.

15. Gressner OA, Schifflers MC, Kim P, Heuts L, Lahme B, Gressner AM. Questioning the role of actinfree Gc-Globulin as actin scavenger in neurodegenerative central nervous system disease: Relationship to S-100B levels and blood-brain barrier function. Clin Chim Acta. 2009;400:86-90.

16. Zhang J, Sokal I, Peskind ER, et al. CSF multianalyte profile distinguishes Alzheimer and Parkinson diseases. Am J Clin Pathol. 2008;129:526-529.

17. Agdeppa ED, Kepe V, Liu J, et al. Binding characteristics of radiofluorinated 6-dialkylamino-2-naphthylethylidene derivatives as positron emission tomography imaging probes for beta-amyloid plaques in Alzheimer's disease. J Neurosci. 2001;21:RC189.

18. Ashford JW, Shih WJ, Coupal J, et al. Single SPECT measures of cerebral cortical perfusion reflect time-index estimation of dementia severity in Alzheimer's disease. J Nucl Med. 2000;41:57-64.

19. Gomme PT, Bertolini J. Therapeutic potential of vitamin D-binding protein. Trends Biotechnol. 2004;22:340-345.

20. Dahl B, Schiødt FV, Ott P, et al. Plasma concentration of Gc-globulin is associated with organ dysfunction and sepsis after injury. Crit Care Med. 2003;31:152-156.

21. Antequera $D$, Vargas $T$, Ugalde C, et al. Cytoplasmic gelsolin increases mitochondrial activity and reduces Abeta burden in a mouse model of Alzheimer's disease. Neurobiol Dis. 2009;36:42-50.

22. Bucki R, Kulakowska A, Byfield FJ, et al. Plasma gelsolin modulates cellular response to sphingosine 1-phosphate. Am J Physiol Cell Physiol. 2010;299:C1516-1523.

23. Bucki R, Byfield FJ, Kulakowska A, et al. Extracellular gelsolin binds lipoteichoic acid and modulates cellular response to proinflammatory bacterial wall components. J Immunol. 2008;181:4936-4944.

24. Bucki R, Georges PC, Espinassous Q, et al. Inactivation of endotoxin by human plasma gelsolin. Biochemistry. 2005;44:9590-9597.

25. Bucki R, Janmey PA. Interaction of the gelsolin-derived antibacterial PBP 10 peptide with lipid bilayers and cell membranes. Antimicrob Agents Chemother. 2006;50:2932-2940.

26. Lukiw WJ, Bazan NG. Survival signalling in Alzheimer's disease. Biochem Soc Trans. 2006:34:1277-1282.

27. Takasugi N, Sasaki T, Ebinuma I, et al. FTY720/fingolimod, a sphingosine analogue, reduces amyloid- $\beta$ production in neurons. PLoS One. 2013;8:e64050.

28. Takasugi N, Sasaki T, Suzuki K, et al. BACE1 activity is modulated by cell-associated sphingosine-1-phosphate. J Neurosci. 2011;31:6850-6857.

29. Fukumoto K, Mizoguchi H, Takeuchi H, et al. Fingolimod increases brain-derived neurotrophic factor levels and ameliorates amyloid $\beta$-induced memory impairment. Behav Brain Res. 2014;268:88-93.

30. Brunkhorst $R$, Vutukuri $R$, Pfeilschifter W. Fingolimod for the treatment of neurological diseases-state of play and future perspectives. Front Cell Neurosci. 2014;8:283.

31. Dutta R, Trapp BD. Mechanisms of neuronal dysfunction and degeneration in multiple sclerosis. Prog Neurobiol. 2011;93:1-12.

32. Trapp BD, Nave KA. Multiple sclerosis: An immune or neurodegenerative disorder? Annu Rev Neurosci. 2008;31:247-269.

33. Nave KA, Trapp BD. Axon-glial signaling and the glial support of axon function. Annu Rev Neurosci. 2008;31:535-561.

34. Micu I, Jiang $Q$, Coderre $E$, et al. NMDA receptors mediate calcium accumulation in myelin during chemical ischaemia. Nature. 2006;439:988-992.

35. Bennett JL, Stüve O. Update on inflammation, neurodegeneration, and immunoregulation in multiple sclerosis: Therapeutic implications. Clin Neuropharmacol. 2009;32:121-132.

36. Gelpi E, Preusser M, Laggner $U$, et al. Inflammatory response in human tick-borne encephalitis: Analysis of postmortem brain tissue. J Neurovirol. 2006;12:322-327.

37. Holick MF. Vitamin D status: Measurement, interpretation, and clinical application. Ann Epidemiol. 2009;19:73-78. 
38. Binkley N, Ramamurthy R, Krueger D. Low vitamin D status: Definition, prevalence, consequences, and correction. Endocrinol Metab Clin North Am. 2010;39:287-301.

39. Littlejohns TJ, Henley WE, Lang IA, et al. Vitamin D and the risk of dementia and Alzheimer disease. Neurology. 2014;83:920-928. 